\title{
Colon Adenocarcinoma Diagnosis in Human Samples by Multicontrast Nonlinear Optical Microscopy of Hematoxylin and Eosin Stained Histological Sections
}

\author{
Javier Adur',2,3, Mariana Bianchi'1, Vitor B. Pelegati³, Silvia Viale1, María F. Izaguirre1, \\ Hernandes F. Carvalho ${ }^{3,4}$, Carlos L. Cesar4,5, Víctor H. Casco ${ }^{1^{*}}$ \\ ${ }^{1}$ Microscopy Laboratory Applied to Molecular and Cellular Studies, School of Engineering, National University \\ of Entre Ríos (UNER), Oro Verde, Argentina \\ ${ }^{2}$ Research and Transfer Center of Entre Ríos (CITER) CONICET-UNER, Oro Verde, Argentina \\ ${ }^{3}$ INFABiC-National Institute of Science and Technology on Photonics Applied to Cell Biology, Campinas, Brazil \\ ${ }^{4}$ Department of Structural and Functional Biology, Institute of Biology, State University of Campinas \\ (UNICAMP), Campinas, Brazil \\ "Biophotonic Group, Optics and Photonics Research Center (CEPOF), Institute of Physics "Gleb Wataghin", \\ State University of Campinas (UNICAMP), Campinas, Brazil \\ Email: vcasco@bioingenieria.edu.ar
}

Received 26 August 2014; revised 20 September 2014; accepted 18 October 2014

Academic Editor: Sibu P. Saha, University of Kentucky, USA

Copyright (C) 2014 by authors and Scientific Research Publishing Inc.

This work is licensed under the Creative Commons Attribution International License (CC BY).

http://creativecommons.org/licenses/by/4.0/

(c) (i)

Open Access

\begin{abstract}
Combined multimodal nonlinear optical (NLO) microscopies were used to detect and quantify morphological changes associated with stroma and epithelial transformation in colon cancer. Our findings provide complementary information about tissue microstructure, displaying distinctive patterns between normal and malignant human colon. Additionally, we have demonstrated the usefulness of using fixed tissues for the disease diagnostic and prognostic. The present work provides a framework for using NLO techniques as a clinical diagnostic tool for human colon cancer. NLO metrics could be applied to other disorders, which are characterized by abnormal cell proliferation and collagen assembly.
\end{abstract}

${ }^{*}$ Corresponding author.

How to cite this paper: Adur, J., et al. (2014) Colon Adenocarcinoma Diagnosis in Human Samples by Multicontrast Nonlinear Optical Microscopy of Hematoxylin and Eosin Stained Histological Sections. Journal of Cancer Therapy, 5, $1259-1269$. http://dx.doi.org/10.4236/jct.2014.513127 
Keywords

\section{Nonlinear Microscopy, Nonlinear Multicontrast, Histological Sections, Human Colon Cancer}

\section{Introduction}

Colon cancer is one of the most widespread cancers in the western world, ranking third worldwide in frequency of incidence after lung and breast cancers [1]. About 96\% of all colorectal cancers are adenocarcinomas; these originate from the epithelial lining of glandular tissue in the colonic mucosa. They typically evolve from benign neoplasms known as adenomatous polyps, through the adenoma-carcinoma sequence [2]. Even though these adenocarcinomas could be curable when they are early detected and treated, many of them can be undiagnosed or misdiagnosed. Therefore, the developments of more precise early diagnostic methods before the growth of the tumor are necessary to improve its prognosis and treatment. To accomplish this, a better understanding of the fundamental changes in epithelium/stroma colonic interface in carcinogenesis is imperative.

In the last decade, the scientific community undertook remarkable efforts to discover both novel and efficient methods such as chromoendoscopy, high resolution and magnification endoscopy, narrow band imaging and autofluorescence imaging for colon cancer diagnosis [3]-[6]. Even with the costs and risks associated, the endoscopic biopsy hematoxylin and eosin stained (H\&E) is still the gold standard for local screening [7]. As an alternative, non-invasive approaches became promising for colon cancer detection. Examples of these techniques are fluorescence spectroscopy, Raman spectroscopy, and non-linear optical (NLO) microscopies, such as two-photon imaging, second harmonic generation (SHG) and third harmonic generation (THG) microscopies [8]-[12].

NLO signals are hugely enhanced by the use of femtosecond pulsed lasers and happen strongly only at the objective focus [13]. While TPEF provides functional information about molecules [14], SHG and THG are endogenous signals that can be used to image subcellular structures and interfaces organization [15] [16]. SHG has proved to be an excellent approach to observe collagen fiber network in extracellular matrices and THG highlights the cell nucleus in a background free image.

The fact that the NLO microscopy can be performed on unstained thick specimens, is the main driving force for its use in the study of both ex vivo and in vivo biological process. However, we have recently demonstrated that NLO microscopy is also an important tool for routinely stained biopsy samples [17]. One very important reason is that long time-scale processes in the range of years can be followed using an already built library of H\&E stained slides of cancer samples. If a new microscopy technique can see new features on those stored materials then, it would be possible to make a retrospective study of long time-scale biological processes. The calibration of a new technique against the gold standard is also an excellent reason to use fixed samples, especially when the chance of uncover extra information not apparent in the H\&E sections is available. In addition, the NLO microscopy could allow the automatization of the analysis in clinical pathology, enhancing the productivity in this field.

Therefore, the aim of this study was to show how the combined use of these three techniques is very useful for cancer studies. Our results show that the multicontrast gives additional insight into the tissue under investigation. The study provides the basis for future applications of nonlinear multicontrast microscopy for histological investigations and presents a first approximation on the discriminating capabilities of this kind of analysis, as well as a better understanding of the different biomarkers for formalin-fixed biopsies.

\section{Material and Methods}

\subsection{Colon Biopsies}

The research protocol was approved by the ethics committee and all procedures were in accordance with the Declaration of Helsinki and the ethical principles of the medical community. Colon biopsies were obtained from paraffin tissue blocks of the Silvia Viale's Laboratory of Pathology and Cytology (Paraná, ER, Argentina). These blocks were previously prepared following standard histological procedure. Afterwards, specimens were sectioned in $4-\mu \mathrm{m}$ and $30-\mu \mathrm{m}$ thick slices and mounted on slides. Tissue sections H\&E-stained were examined for TPEF, SHG and THG experiments using standard techniques [16] [17]. Furthermore, each H\&E-stained tis- 
sue section was evaluated by the same certified pathologist to avoid bias in the biopsy analysis as well as to promote a homogeneous histological classification of the tumors. Colonic adenocarcinomas from 7 biopsies $(\mathrm{n}=$ 7) and their corresponding normal colon region ( $n=7$, matched controls) were analyzed.

\subsection{Multimodal NLO Setup}

The experimental multimodal setup employed was built at the National Institute of Science and Technology on Photonics Applied to Cell Biology (INFABIC) at the State University of Campinas consists of a Zeiss LSM 780-NLO confocal scanner on an Axio Observer Z.1 microscope (Carl Zeiss AG, Germany). The system is mainly composed of three parts: the laser source and the optical system on the bench, the scanner and microscope, and transmitted and reflected detectors. A schematic view of the system is depicted in Figure 1.

TPEF, SHG, THG, signals were registered exciting the tissues with a Ti:Sapphire Mai Tai HP Spectra-Physics (Irvine, USA) laser, which provides 100 femtoseconds (fs) pulses from 690 to $1040 \mathrm{~nm}$, with a repetition rate of $80 \mathrm{MHz}$ and power from 1 to $3.5 \mathrm{~W}$. It was equipped with a Deep See for group velocity dispersion compensation. The fs beam was coupled to the scan head through an acousto-optic modulator (AOM) for a fast intensity control and with a collimating telescope $(\mathrm{T})$ to adjust both the beam diameter and to fill the objective backaperture of the objective and the beam focus position on the microscope focal plane. All images were acquired with an EC Plan-Neofluar 40×/1.30 oil immersion objective (Carl Zeiss, Germany). TPEF, SHG and THG signals were collected in the transmission mode but TPEF were also collected in the backscattering mode. THG was collected separately from TPEF and SHG signals to avoid THG UV light blocking by the optical elements of the microscope. To carry out this procedure, a special detector support was developed to hold it, as close to the sample as possible, without touching it, but with enough space for the optical filters. The TPEF images collected both in the transmission and backscattering mode, were compared to be sure that the sample did not move after THG acquisition procedure. Sensitive Non Descanned Detector (NDD) and an external R3896 photomultiplier tubes (PMTs) (Hamamatsu Photonics, Hamamatsu City, Japan) were used to detect TPEF/SHG and THG

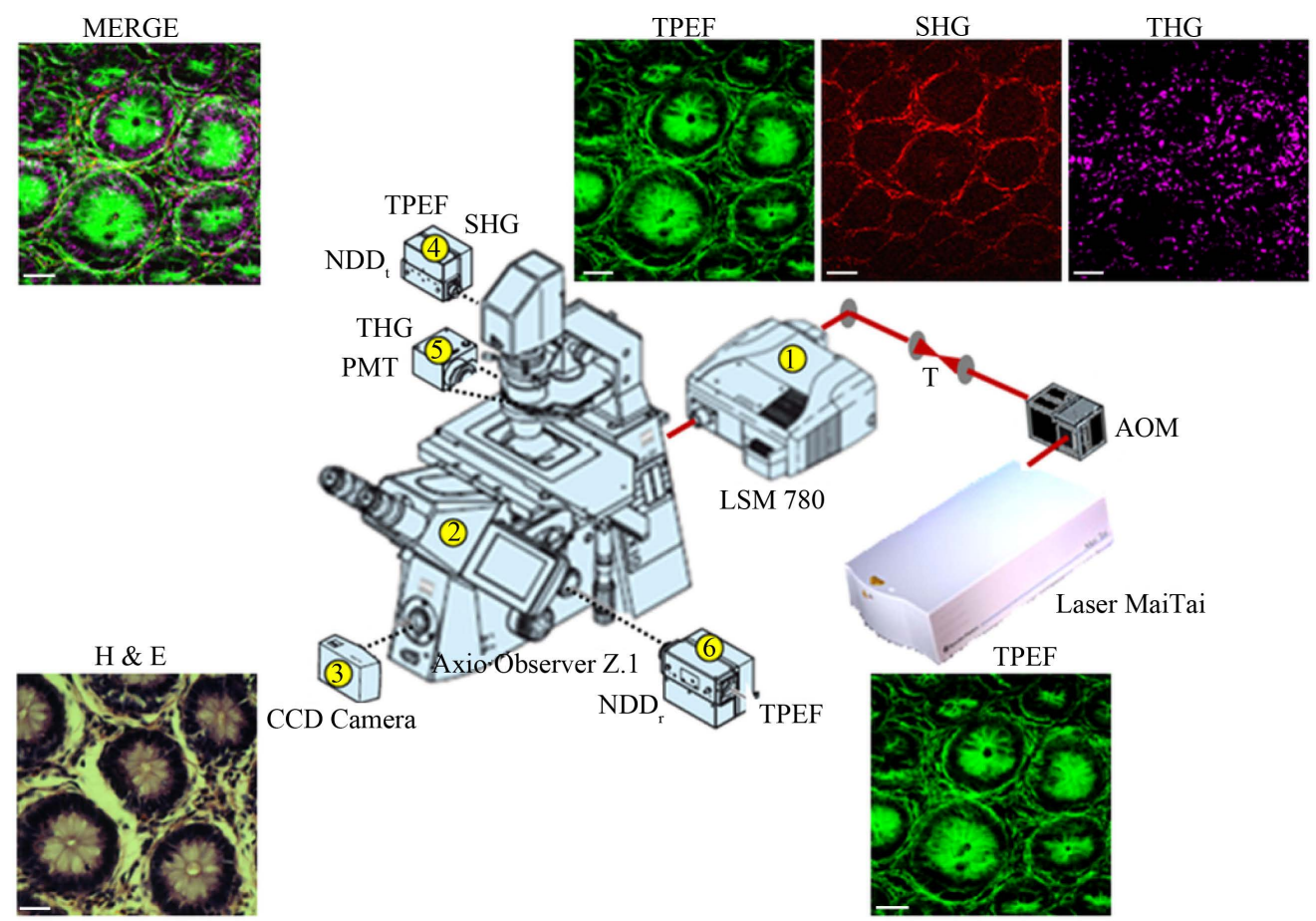

Figure 1. Schematic view of multimodal NLO setup. In black letters principal components (laser, microscope, and detectors) and in color letters different nonlinear signal collected are indicated. (1) Scan head LSM 780; (2) Inverted Axio Observer Z.1 microscope; (3) CCD camera; (4) transmitted NND detector; (5) external PMT detector; and (6) reflected NDD detector. AOM: acousto-optic modulator, T: telescope, CCD: charge-coupled device. TPEF, SHG, and THG collected in transmission mode (upper). $\mathrm{H} \& \mathrm{E}$ and TPEF collected in reflection mode (below). Scale bar $=20 \mu \mathrm{m}$. 
respectively. For comparison, wide field standard H\&E images were acquired with a charge-coupled device (CCD) color camera (DFK 31AF03, The Imaging Source, Germany), placed in the front port of the microscope (Figure 1).

\subsection{Image Acquisition}

Signal images were acquired with $940 \mathrm{~nm}$ for TPEF, SHG, and THG excitations. According to our previous experiences in breast and ovary tumor tissues [18] [19], $940 \mathrm{~nm}$ laser beam can excite the three signals TPEF, SHG, and THG at the same time; generating a TPEF at $>500 \mathrm{~nm}$, SHG at $470 \mathrm{~nm}$, and THG at $313 \mathrm{~nm}$ (average power in tissue sample less than $15 \mathrm{~mW}$ ). Transmitted signals were detected after blocking the SP690 filter to prevent laser light and using the following filter cube configurations in NDD detectors: 1) dichroic mirror 490 LP and 485 SP filter for SHG; 2) 520 - 560 BP filter for TPEF; 3) U340 $\pm 30 \mathrm{~nm}$ colored glass filter (Hoya Corporation) for THG, which was located in front of the external PMT detector. TPEF reflected signals were also collected after blocking the SP 690 filter to prevent laser light and using the following filter cube configuration in NDD detectors: 520 - 560 BP filter.

All images were acquired with $512 \times 512$ pixel spatial resolution, using a pixel dwell time of $6.30 \mu$ s with total scanning time of the order of $60 \mathrm{~s}$, after a 16 frame averaging. Remaining parameters, such as detector gain and offset, were maintained constant between different samples to enable comparative analysis. Images (pseudo-green TPEF, pseudo-red SHG, and pseudomagenta THG) were combined into a single image for visualization using ZEN 2009 Light Edition software (Carl Zeiss MicroImaging GmbH, Germany) (Figure 1).

\subsection{Quantification of Nonlinear Images}

Data from SHG and THG images were quantified using Image 1.45 software (NIH). Based on THG images, quantification of morphometric (area and circularity) and densitometric (kurtosis) features of nuclei were performed. At least 50 cell nuclei were measured in different intestinal crypt of each image analyzed and data were stored automatically into different data files. To carry out this last study the ImageJ "Particle Analysis-Nucleus Counter" plug-in was used. The plug-in performs an automatic threshold and displays the outcome in a table where each nucleus can be identified. Result table will present all features previously selected in "Analyze-Set Measurements" menu. Prior to analysis, a standardized gray-tone scale was used with a range between "0" (black) and "256" (white).

From SHG images, intensity levels and collagen fiber angles were measured. In the first case, a method similar to that previously reported was employed [20]. Briefly, a square region of interest (ROI) $150 \times 150$ pixels was drawn and positioned in the image. The square coordinates were saved as a ROI file in ImageJ. This positioning was repeated so that 9 non-overlapping ROIs were generated and saved. This ROIs array was used together to assess the entire SHG image on a ROI basis in a consistent manner for each sample in the array. Using the multi-measure plugin, the 9 ROI files were opened and visualized as an overlapped image, and separated standard measurements from each of the threshold ROIs were obtained. From this data the area fraction (the percentage of the ROI area that is above the SHG threshold and is essentially a measure of SHG prevalence) was quantified. The collagen fibers angles were measured using the tangent of the epithelium-stroma interface, as was previously reported [20] [21]. Using the ImageJ angle tool, the angle was defined by three points as following: the first one is a point along the fibril, the second is the fibril extreme closest to the tumor epithelium (edge); and the third one any point that connected to the first draws a line parallel to the epithelium. In total, 150 angles were measured for each tumor type.

\subsection{Statistical Analysis}

We performed $t$-testing for two-group comparisons. The level of significance employed was: significant $\left({ }^{*}\right) p<$ 0.05 and very significant $\left({ }^{* *}\right) p<0.01$. Data were analyzed with SPSS 10.0 software.

\section{Results}

\subsection{Comparison of Nonlinear and H\&E-Stained Images of Human Colon}

NLO images clearly demonstrate the circular arrangement pattern of control colonic crypts registered from crypt-cross sections, characterized by epithelial columnar cells and interspersed goblet cells (Figure 2). Green 
TPEF signal corresponds to eosin fluorescence, which is usually weak in nuclear regions but strong at the cell cytoplasm and stroma collagen fibers. TPEF revealed the typical foveolar pattern of colon mucosa glands, displaying crypts with rounded-luminal openings (Figure 2(B)). SHG (red) traced specifically the collagen scaffold within lamina propria (Figure 2(C)) and THG (magenta) highlighted the nuclei of mucosa epithelial cells (Figure 2(D)).

According to our knowledge, these are the first study showing the integration of TPEF, SHG and THG in human colon biopsies. Highly valuable, structural information revealed by each nonlinear contrast mechanism can be isolated and analyzed separately, while their superposition allows a better comparison and understanding of the spatial organization of the tissue (Figure 2(E)).

\subsection{Colonic Tissue Evaluation in H\&E-Stained Sections by SHG and THG Microscopy}

Evaluation of colonic tissue by SHG (Figure 3) and THG (Figure 4) microscopies rapidly and clearly allows differentiating between adenocarcinoma and normal tissue states. Whereas individual crypts were easily identified with TPEF, the connective tissue between them was detected via SHG of collagen (Figure 3(A) and Figure 3(B)). In tumor tissue, SHG images highlights changes in the surrounding fibrous stroma. The quantification of collagen prevalence over the tumor tissues SHG images results in a significant difference compared to the normal colon (Figure 3(C)), demonstrating an increased collagen density in tumor stroma. While normal colon exhibits collagen fiber bundles approximately parallel to the epithelium-stroma interface, adenocarcinomas have thicker and nonparallel collagen fibers located at certain angles respect to such interface (Figure 3(B)). Quantitative

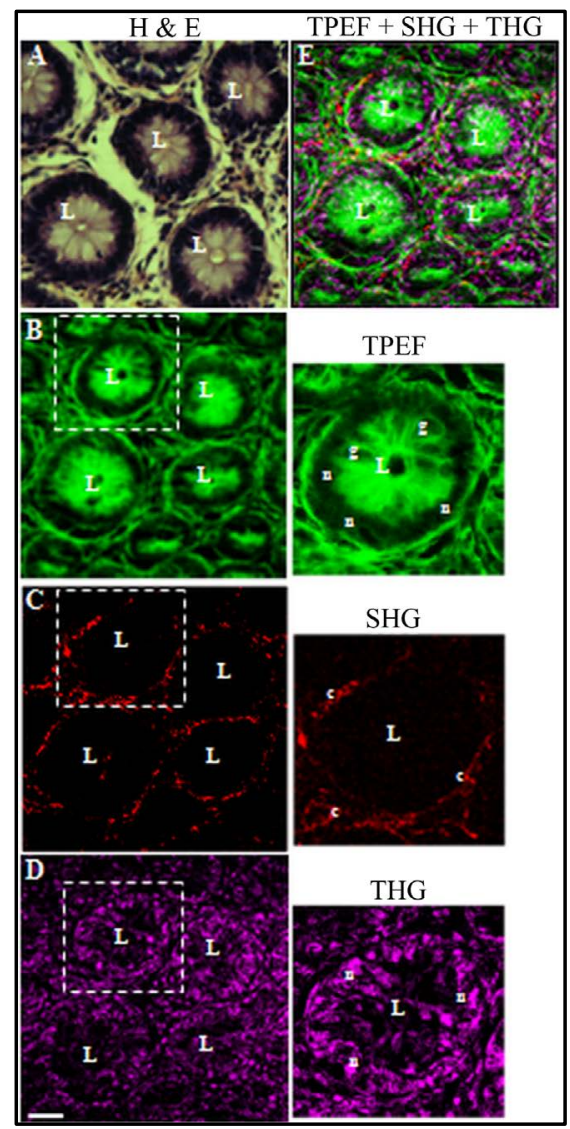

Figure 2. Multicontrast representative in face imaging (512 × 512 pixels) of normal colon tissue. (A) Typical histological H\&E images used for comparison; (B)-(E) Normal colon tissue and in the right individual crypt (white ROI) showed at higher magnification; (B)-(D) TPEF, SHG, and THG images of H\&E-stained sections and (E) merge image. The principal contrasts produced for each technique are, in green: two-photon excited fluorescence (TPEF); in red: second harmonic generation (SHG); in magenta: third harmonic generation (THG). Structures, L: luminal crypt orifice, g: goblet cells, n: nucleus, c: collagen fibers. Scale bar $=20 \mu \mathrm{m}$. 

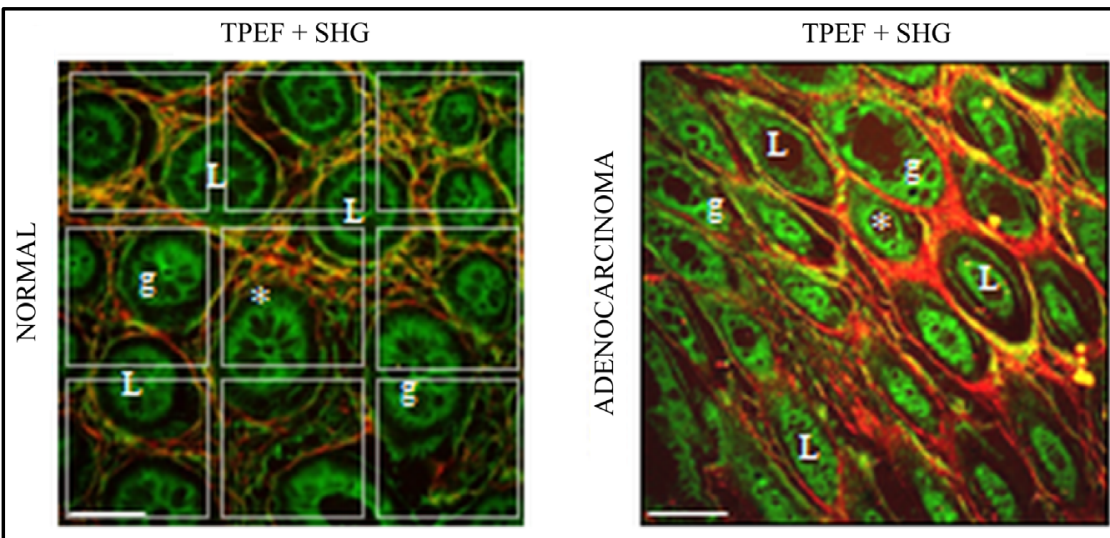

(A)
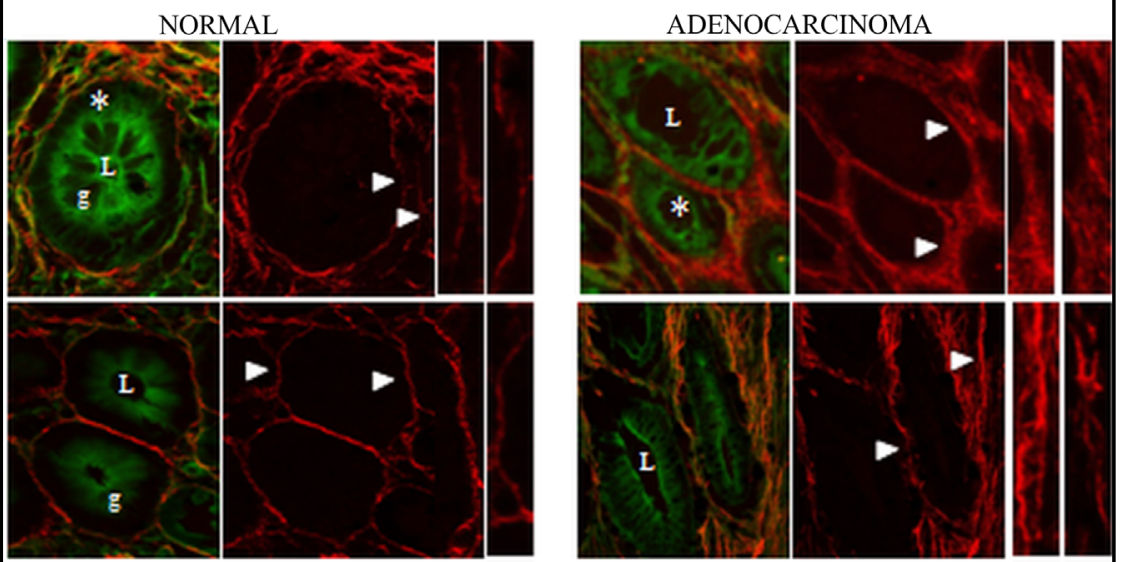

(B)
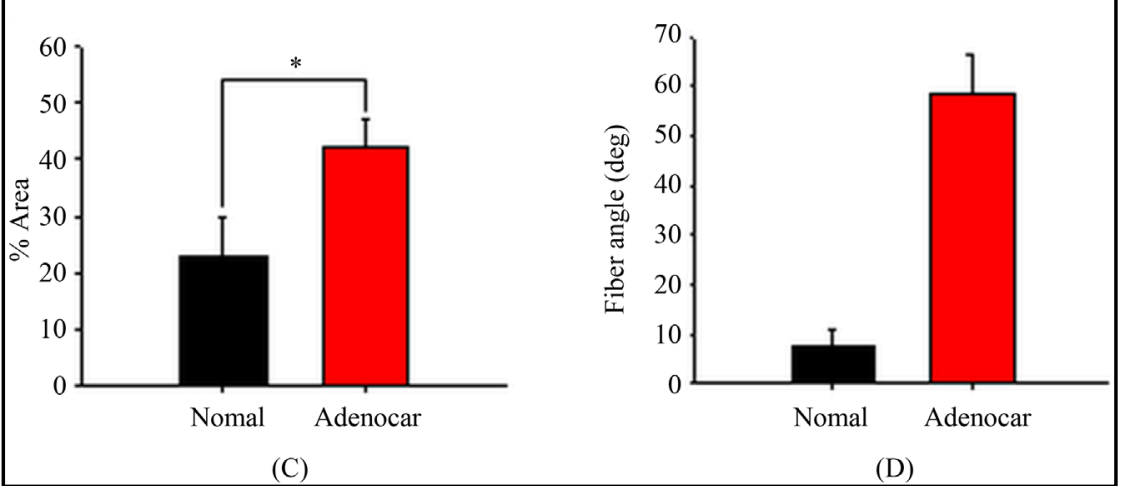

Figure 3. Representative TPEF (green) + SHG (red) in face imaging at a two-photon excitation wavelength of $940 \mathrm{~nm}$ of normal and adenocarcinoma colonic mucosa. (A) TPEF + SHG $(512 \times 512$ pixels $)$ image. Normal image shows the 9 representative white ROI $(150 \times 150$ pixels) where quantification of collagen content was performed. White asterisk ( $\left(^{*}\right)$ indicate individual crypt selected to analyze collagen fiber angle. Scale bar = $50 \mu \mathrm{m}$. (B) Magnified individual crypt selected from (see ${ }^{*}$ in (A)). Two images from different biopsies are displayed. Collagen fibers angle were quantified from these images. Specific fibers (white arrowhead) are enlarged to discriminate the fiber morphology. Structures in (A) and (B), L: luminal crypt orifice, g: goblet cells, n: nucleus, c: collagen fibers. (C) Area quantification with $n=117$ and $n=81$ ( $n=$ number of ROI $(150 \times 150$ pixels) for normal and adenocarcinoma tissues respectively. (D) Fiber angle quantification with $n=150$ and $n=135$ ( $n=$ number of collagen fibers) for normal and adenocarcinoma samples respectively. Each bar represents the mean \pm S.D. ${ }^{*} p<0.05$ according to $t$-test. 

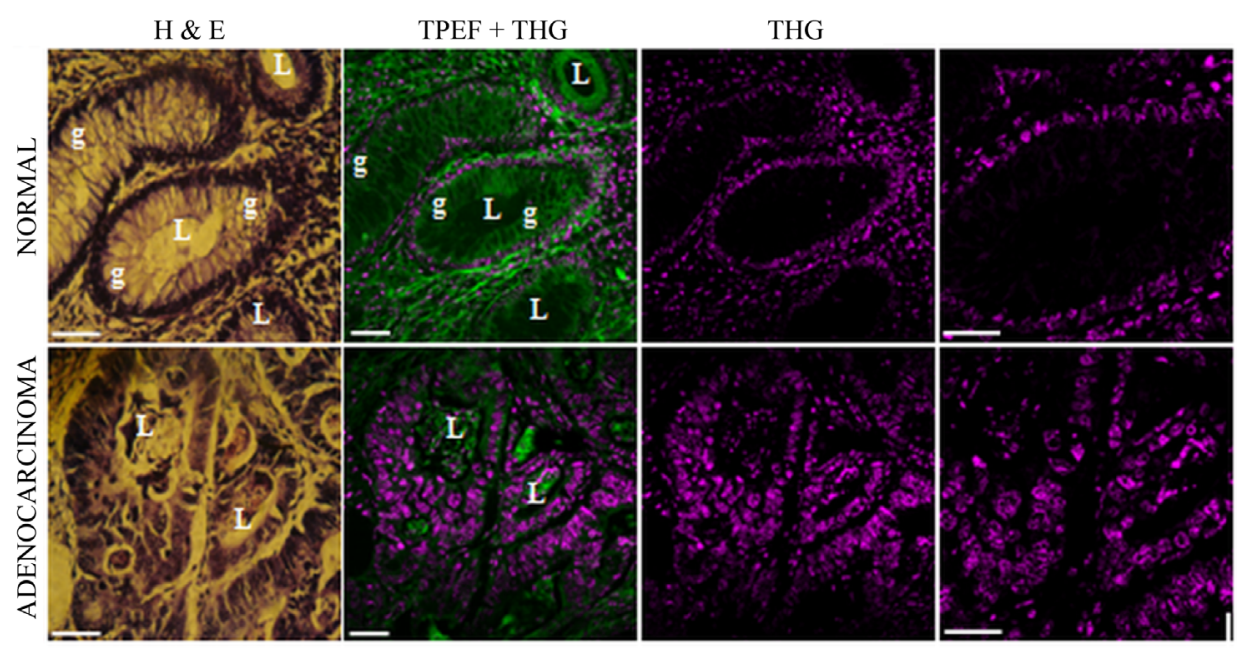

(A)
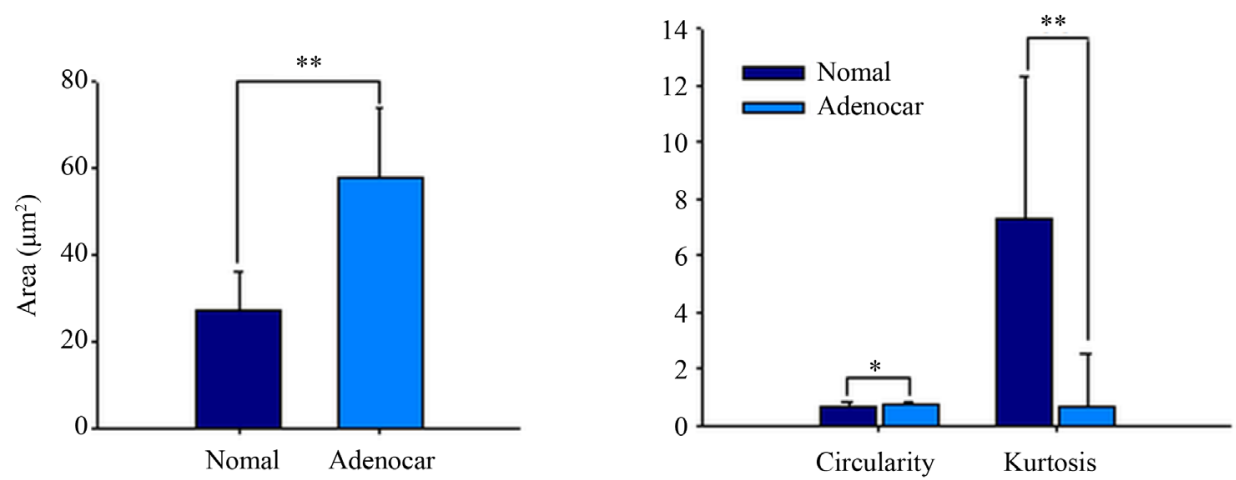

(B)

Figure 4. (A) Representative TPEF (green) + THG (magenta) in face imaging (512 × 512 pixels) at two-photon excitation wavelength of $940 \mathrm{~nm}$ of normal and adenocarcinoma colonic mucosa. L: luminal crypt orifice, g: goblet cells. All scale bar $=50 \mu \mathrm{m}$. (B) Area, circularity and kurtosis quantification with $n=580$ and $n=560$ ( $n=$ number of nucleus) for normal and adenocarcinoma samples respectively. Each bar represents the mean \pm S.D. ${ }^{*} p<0.05$ and ${ }^{* *} p<0.001$, respectively according to $t$-test.

analysis showed that the angles of collagen fibers in normal colon are lower than $11^{\circ}$ whereas adenocarcinomas have angles higher than $50^{\circ}$ (Figure 3(D)).

Even though the analysis must be performed in classical H\&E images by a well-trained pathologist, THG images allow detecting selectively just the nuclei, making easier the digitalization process. Enlarged and irregular shaped tumor cells exhibit increased nucleus-to-cytoplasm ratio (Figure 4(A)), area and circularity are significantly higher, and gray value distribution is broader (lower kurtosis value) in tumor cells nuclei due to a considerable heterogeneity, showing distinct areas with considerable brightness and darkness (Figure 4(B)). Additionally, a very important point to highlight in comparison to classical histological techniques is the potential benefit of 3D capabilities of the proposed NLO techniques. Figure 5 shows three-dimensional representations (single or combined) that allows the visualization of indistinguishable characteristics in classical two-dimensional procedures. Maximum projection 3D representation using THG images (Figure 5(A) and Figure 5(B)) clearly demonstrated the adenocarcinoma architectural change, in which the circular arrangements of each colonic crypt and the spacing between cells have been lost. Also, an attenuation of goblet cell/crypt density and mucin depletion were easily distinguished (Figure 5(A)). Moreover, using a three-dimensional SHG representation it was possible to observe the inclination and invasiveness of collagen fibers of adenocarcinomas compared to normal colon (Figure 5(C)). It is worth pointing out that these features (presence and angle of collagen fibers) are not easily visible in H\&E-stained sections. 


\section{NORMAL}

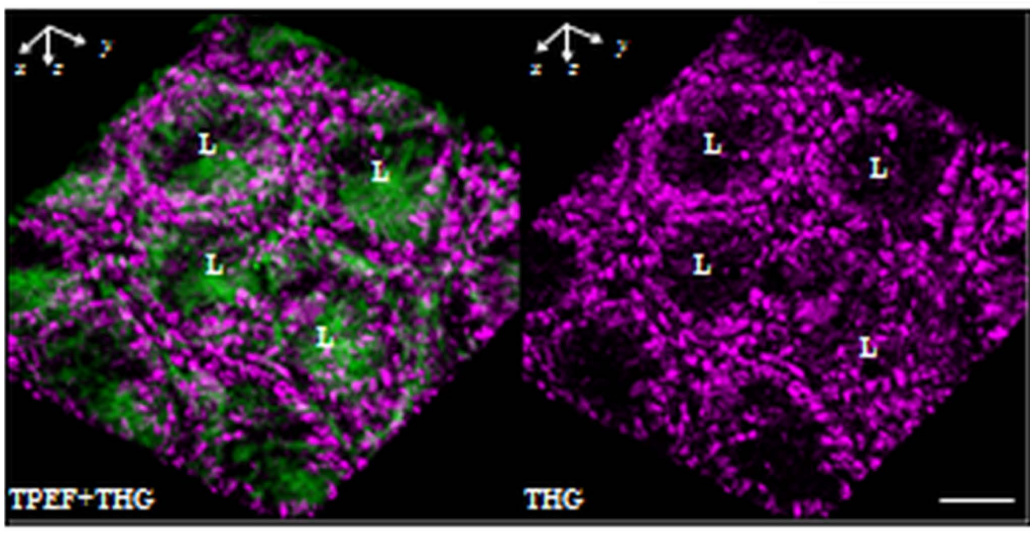

(A)

ADENOCARCINOMA

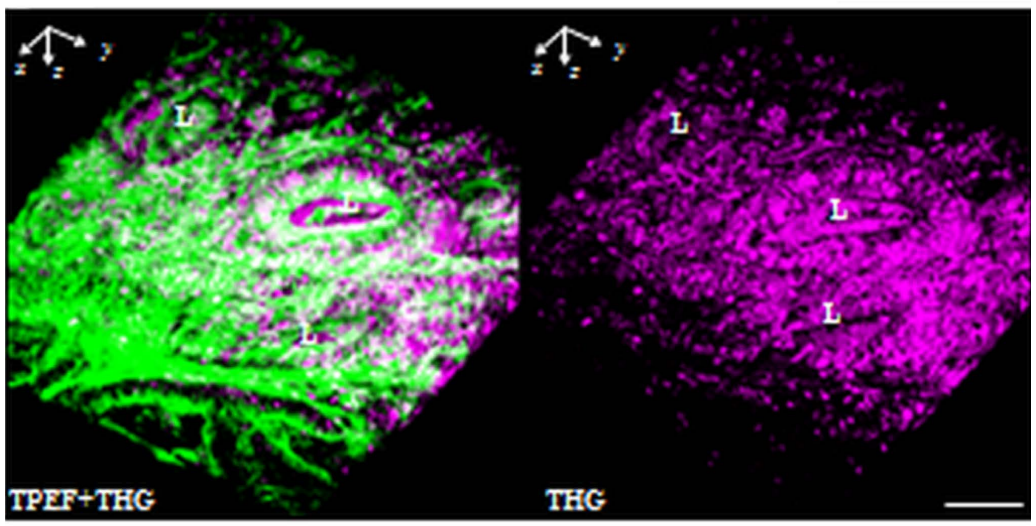

(B)

NORMAL

ADENOCARCINOMA

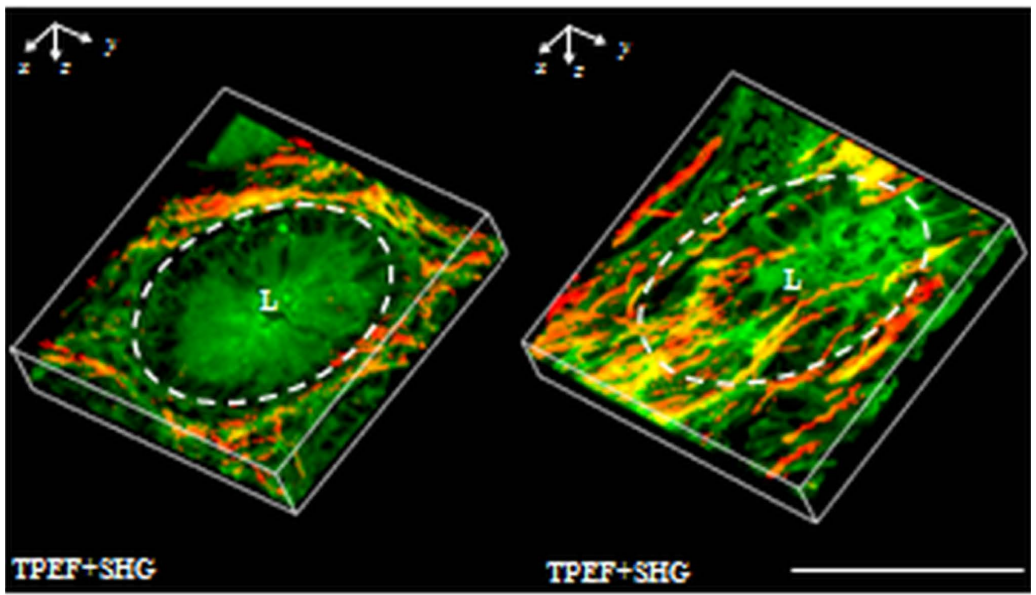

(C)

Figure 5. Maximum projection of 60 images separated $0.5 \mu \mathrm{m}$ each $(512 \times 512 \times$ $60)$. (A) normal and (B) adenorcarcinomas colonic tissues. At left side, TPEF + THG representations and on the right, only THG representations of nuclei cells. C) Maximum projection (TPEF + SHG) of individual crypts. 60 images and 50 images separated $0.5 \mu \mathrm{m}$ each in normal and adenocarcinomas samples respectively. Epithelial/stromal interface is indicated (white outline). L: luminal crypt orifice, g: goblet cells. All scale bar $=50 \mu \mathrm{m}$. 


\section{Discussion}

The present report demonstrates that the combination of different NLO techniques may be used, not only to differentiate cancerous from normal colon, but also to obtain detailed information on tissue architecture and features of its components.

It's known that hematoxylin stain can selectively enhance nuclear THG yield at cell nuclei. The absorption spectrum of hematoxylin exhibits a broad range of absorption at the visible light band, achieving the two- and three-photon resonance enhancement criteria [16] [22]. The THG observed in H- and H\&E-stained tissue are stronger than in dried precipitates, most probably due to differences in refractive index and nonlinear susceptibility of the tissue environment, and interaction of the dyes with chromatin [16]. The verified enhancement of THG signals, originated almost exclusively from the nuclear regions, is coincident with these observations. H\&E stain was used to register TPEF, SHG and THG, because our and other previous reports have demonstrated that H\&E stain is not only useful to generate TPFE signal [23] but also enhance the THG signal without any alteration of the SHG signal [16] [18] [22]. Although there are some evidences suggesting that tissue postprocessing procedures may affect how intrinsic fluorescent signals respond under multiphoton excitation, previous work have proved that structural proteins such as collagen and elastin are little affected by post-processing procedures [16] [24] [25].

Interestingly, the outcome obtained from TPEF and SHG signals (Figure 2(B) and Figure 2(C)) are in line with the analyses on fresh tissues obtained by other researchers [7] [12] [26] [27], showing the potential of these tools over archive slides. Furthermore, a recent work has used SHG signal from H\&E-stained slices to discriminate between high grade dysplasia and cancer from normal human colonic mucosa [28]. This work clearly shows significant changes of collagen fiber morphology, orientation and density in colon tumor tissue. The present report suggests that collagen fiber inclination angles are an important factor in tumor progression. In agreement with our results, previous reports in human colon and other tissues suggest that the epithelial cells preferentially invade tissues where the collagen fibers became perpendicularly aligned, instead of randomly arranged ones [12] [18] [20] [28].

The fact that NLO microscopy can see image selective specimen properties in 2D and 3D mode, makes it easier to digitally process the images opens the possibility to automatically discriminate between the different types of disease. Integration of many techniques would improve the differential diagnosis power. Also, these properties of NLO images can be very useful to detect abnormal mucosa and stroma configurations in early stages of the disease. Recently, working with mouse CCR model, we observed that SHG signals from collagen fibrils during tumor progression shows differences that might serve as an early biomarker of colon cancer risk (manuscript in preparation).

\section{Conclusion}

In conclusion, the use of several NLO microscopy approaches, especially when combined, can reveal information not distinguishable in H\&E stained sections under classical bright field approaches. Cell nucleus size and shape, tightness and disposition changes of collagen fibers are parameters that can be consistently quantified, allowing them to predict a huge clinical potential. These imaging modalities may also find future application in the clinical setting considering the emerging potential of non-linear microscopy to become an in vivo or ex vivo diagnostic tool.

\section{Acknowledgements}

The authors are grateful to FAPESP Process No. 2011/51591-3, PICTO ANPCYT-UNER-2009-209 and PIDUNER N ${ }^{\circ} 6116$. This work is also linked with the CEPOF (Optics and Photonics Research Center, FAPESP) and National Institute of Photonics Applied to Cell Biology (INFABIC).

\section{References}

[1] Jemal, A., Bray, F., Center, M.M., Ferlay, J., Ward, E. and Forman, D. (2011) Global Cancer Statistics. CA: A Cancer Journal for Clinicians, 61, 69-90. http://dx.doi.org/10.3322/caac.20107

[2] Taylor, J.C., Kendall, C.A., Stone, N. and Cook, T.A. (2007) Optical Adjuncts for Enhanced Colonoscopic Diagnosis. British Journal of Surgery, 94, 6-16. http://dx.doi.org/10.1002/bjs.5628 
[3] Rex, D.K. (2007) Colonoscopy: The Dominant and Preferred Colorectal Cancer Screening Strategy in the United States. Mayo Clinic Proceedings, 82, 662-664. http://dx.doi.org/10.1016/S0025-6196(11)61183-X

[4] Lopes, P.C., Moreira, J.A., Almeida, A., Esteves, A., Gregora, I., Ledinsky, M., Lopes, J.M., Henrique, R. and Oliveira, A. (2011) Discriminating Adenocarcinoma from Normal Colonic Mucosa through Deconvolution of Raman Spectra. Journal of Biomedical Optics, 16, Article ID: 127001.

[5] Banerjee, B., Renkoski, T., Graves, L.R., Rial, N.S., Tsikitis, V.L., Nfonsam, V., Pugh, J., Tiwari, P., Gavini, H. and Utzinger, U. (2012) Tryptophan Autofluorescence Imaging of Neoplasms of the Human Colon. Journal of Biomedical Optics, 17, Article ID: 016003. http://dx.doi.org/10.1117/1.JBO.17.1.016003

[6] Imaizumi, K., Harada, Y., Wakabayashi, N., Yamaoka, Y., Konishi, H., Dai, P., Tanaka, H. and Takamatsu, T. (2012) Dual-Wavelength Excitation of Mucosal Autofluorescence for Precise Detection of Diminutive Colonic Adenomas. Gastrointestinal Endoscopy, 75, 110-117. http://dx.doi.org/10.1016/j.gie.2011.08.012

[7] Cicchi, R., Sturiale, A., Nesi, G., Tonelli, F. and Pavone, F.S. (2012) Two-Photon Imaging and Spectroscopy of Fresh Human Colon Biopsies. Proceedings of SPIE, 8226. http://dx.doi.org/10.1117/12.907822

[8] Tadrous, P.J. (2000) Methods for Imaging the Structure and Function of Living Tissues and Cells: Fluorescence Lifetime Imaging. Journal of Pathology, 191, 229-234.

http://dx.doi.org/10.1002/1096-9896(200007)191:3<229::AID-PATH623>3.0.CO;2-B

[9] Tadrous, P.J., Siegel, J., French, P.M., Shousha, S., Lalani, E.-N. and Stamp, G.W. (2003) Fluorescence Lifetime Imaging of Unstained Tissues: Early Results in Human Breast Cancer. Journal of Pathology, 199, 309-317. http://dx.doi.org/10.1002/path.1286

[10] Carriles, R., Schafer, D.N., Sheetz, K.E., Field, J.J., Cisek, R., Barzda, V., Sylvester, A.W. and Squier, J.A. (2009) Invited Review Article: Imaging Techniques for Harmonic and Multiphoton Absorption Fluorescence Microscopy. Review of Scientific Instruments, 8, Article ID: 081101.

[11] Campagnola, P.J. and Dong, C.Y. (2011) Second Harmonic Generation Microscopy: Principles and Applications to Disease Diagnosis. Laser \& Photonics Reviews, 5, 13-26. http://dx.doi.org/10.1002/lpor.200910024

[12] Zhuo, S., Zhu, X., Wu, G., Chen, J. and Xie, S. (2011) Quantitative Biomarkers of Colonic Dysplasia Based on Intrinsic Second-Harmonic Generation Signal. Journal of Biomedical Optics, 16, Article ID: 120501. http://dx.doi.org/10.1117/1.3659715

[13] Débarre, D., Supatto, W., Pena, A.M., Fabre, A., Tordjmann, T., Combettes, L., Schanne-Klein, M.C. and Beaurepaire, E. (2006) Imaging Lipid Bodies in Cells and Tissues Using Third-Harmonic Generation Microscopy. Nature Methods, 3, 47-53. http://dx.doi.org/10.1038/nmeth813

[14] Zipfel, W.R., Williams, R.M. and Webb, W.W. (2003) Nonlinear Magic: Multiphoton Microscopy in the Biosciences. Nature Biotechnology, 21, 1369-1377. http://dx.doi.org/10.1038/nbt899

[15] Cicchi, R., Massi, D., Sestini, S., Carli, P., De Giorgi, V., Lotti, T. and Pavone, F.S. (2007) Multidimensional NonLinear Laser Imaging of Basal Cell Carcinoma. Optics Express, 15, 10135-10148. http://dx.doi.org/10.1364/OE.15.010135

[16] Tuer, A., Tokarz, D., Prent, N., Cisek, R., Alami, J., Dumont, D.J., Bakueva, L., Rowlands, J. and Barzda, V. (2010) Nonlinear Multicontrast Microscopy of Hematoxylin-and-Eosin-Stained Histological Sections. Journal of Biomedical Optics, 15, Article ID: 026018. http://dx.doi.org/10.1117/1.3382908

[17] Adur, J., Pelegati, V.B., de Thomaz, A.A., Baratti, M.O., Almeida, D.B., Andrade, L.A., Bottcher-Luiz, F., Carvalho, H.F. and Cesar, C.L. (2012) Optical Biomarkers of Serous and Mucinous Human Ovarian Tumor Assessed with Nonlinear Optics Microscopies. PLoS ONE, 7, e47007. http://dx.doi.org/10.1371/journal.pone.0047007

[18] Adur, J., Pelegati, V.B., Costa, L.F., Pietro, L., de Thomaz, A.A., Almeida, D.B., Bottcher-Luiz, F., Andrade, L.A. and Cesar, C.L. (2011) Recognition of Serous Ovarian Tumors in Human Samples by Multimodal Nonlinear Optical Microscopy. Journal of Biomedical Optics, 16, Article ID: 096017. http://dx.doi.org/10.1117/1.3626575

[19] Adur, J., Pelegati, V.B., de Thomaz, A.A., D’Souza-Li, L., Assunção Mdo, C., Bottcher-Luiz, F., Andrade, L.A. and Cesar, C.L. (2012) Quantitative Changes in Human Epithelial Cancers and Osteogenesis Imperfecta Disease Detected Using Nonlinear Multicontrast Microscopy. Journal of Biomedical Optics, 17, Article ID: 081407. http://dx.doi.org/10.1117/1.JBO.17.8.081407

[20] Conklin, M.W., Eickhoff, J.C., Riching, K.M., Pehlke, C.A., Eliceiri, K.W., Provenzano, P.P., Friedl, A. and Keely, P.J. (2011) Aligned Collagen Is a Prognostic Signature for Survival in Human Breast Carcinoma. The American Journal of Pathology, 178, 1221-1232. http://dx.doi.org/10.1016/j.ajpath.2010.11.076

[21] Provenzano, P.P., Eliceiri, K.W., Campbell, J.M., Inman, D.R., White, J.G. and Keely, P.J. (2006) Collagen Reorganization at the Tumor-Stromal Interface Facilitates Local Invasion. BMC Medicine, 4, 38. http://dx.doi.org/10.1186/1741-7015-4-38

[22] Yu, C.H., Tai, S.P., Kung, C.T., Lee, W.J., Chan, Y.F., Liu, H.L., Lyu, J.Y. and Sun, C.K. (2008) Molecular Third- 
Harmonic-Generation Microscopy through Resonance Enhancement with Absorbing Dye. Optics Letters, 33, 387-389. http://dx.doi.org/10.1364/OL.33.000387

[23] De Carvalho, H.F. and Taboga, S.R. (1996) The Applicability of Hematoxylin-Eosin Staining plus Fluorescence or Confocal Laser Scanning Microscopy to the Study of Elastic Fibers in Cartilages. Comptes Rendus de l'Académie des Sciences-Series III-Sciences de la Vie, 319, 991-996.

[24] Drezek, R., Brookner, C., Pavlova, I., Boiko, I., Malpica, A., Lotan, R., Follen, M. and Richards-Kortum, R. (2001) Autofluorescence Microscopy of Fresh Cervical-Tissue Sections Reveals Alterations in Tissue Biochemistry with Dysplasia. Photochemistry and Photobiology, 73, 636-641. http://dx.doi.org/10.1562/0031-8655(2001)0730636AMOFCT2.0.CO2

[25] Bird, D.K., Yan, L., Vrotsos, K.M., Eliceiri, K.W., Vaughan, E.M., Keely, P.J., White, J.G. and Ramanujam, N. (2005) Metabolic Mapping of MCF10A Human Breast Cells via Multiphoton Fluorescence Lifetime Imaging of the Coenzyme NADH. Cancer Research, 65, 8766-8773. http://dx.doi.org/10.1158/0008-5472.CAN-04-3922

[26] Provenzano, P.P., Rueden, C.T., Trier, S.M., Yan, L., Ponik, S.M., Inman, D.R., Keely, P.J. and Eliceiri, K.W. (2008) Nonlinear Optical Imaging and Spectral-Lifetime Computational Analysis of Endogenous and Exogenous Fluorophores in Breast Cancer. Journal of Biomedical Optics, 13, Article ID: 031220. http://dx.doi.org/10.1117/1.2940365

[27] Cicchi, R., Sturiale, A., Nesi, G., Kapsokalyvas, D., Alemanno, G., Tonelli, F. and Pavone, F.S. (2013) Multiphoton Morpho-Functional Imaging of Healthy Colon Mucosa, Adenomatous Polyp and Adenocarcinoma. Biomedical Optics Express, 4, 1204-1213. http://dx.doi.org/10.1364/BOE.4.00120432

[28] Birk, J.W., Tadros, M., Moezardalan, K., Nadyarnykh, O., Forouhar, F., Anderson, J. and Campagnola, P. (2014) Second Harmonic Generation Imaging Distinguishes Both High-Grade Dysplasia and Cancer from Normal Colonic Mucosa. Digestive Diseases and Sciences, 59, 1529-1534. 
Scientific Research Publishing (SCIRP) is one of the largest Open Access journal publishers. It is currently publishing more than 200 open access, online, peer-reviewed journals covering a wide range of academic disciplines. SCIRP serves the worldwide academic communities and contributes to the progress and application of science with its publication.

Other selected journals from SCIRP are listed as below. Submit your manuscript to us via either submit@scirp.org or Online Submission Portal.
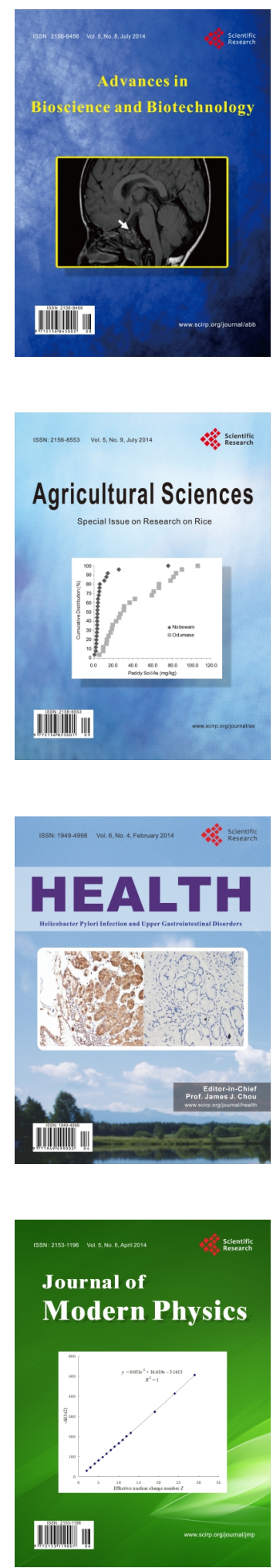
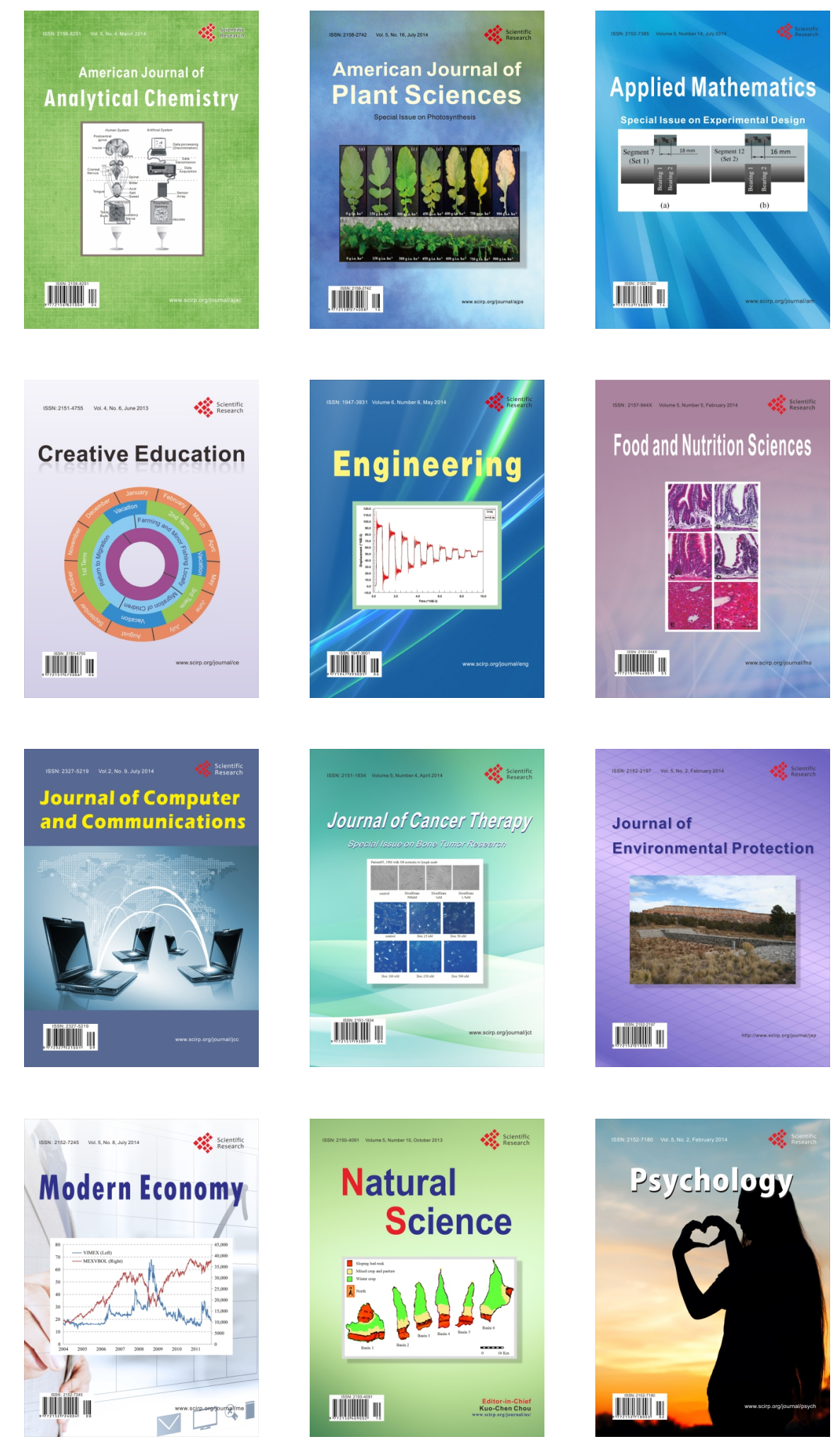